\title{
Special issue for the 2nd International Congress on Advanced Materials
}

\author{
Peter A. Lieberzeit
}

Received: 6 November 2013/Accepted: 9 November 2013/Published online: 11 December 2013

(C) Springer-Verlag Wien 2013

It may be somewhat unexpected to find a special issue of Chemical Monthly dealing with materials science, because the journal is of course especially well known among scientists focusing on synthetic chemistry. However, as the borders between different natural sciences diminish, this is even more so for dividing lines within a specialization such as chemistry. The main challenges in synthetic chemistry are dominated by two driving forces: one is to design novel functional molecules or to find practicable synthetic strategies, especially targeting diagnostics and treatment of diseases; the other is to design novel, high-performance materials to ensure cost-effective and sustainable technological development. The strong interest in the field of developing and designing novel materials leads to a correspondingly large number of conferences on materials science each year. This special issue of Chemical Monthly collects papers presented at the 2nd International Congress on Advanced Materials (AM2013; http://am2013.ujs.edu.cn) held at Jiangsu University, Zhejiang, PR China, from May 16th to 19th, 2013. It brought together almost 600 scientists from China and abroad. The organizing committee of this relatively new conference series consists of an international group of scientists strongly rooted in China, Europe, North America, and Africa and is supported by the International Union of Advanced Materials (http://www.iuam.org) and the Chinese Advanced Materials Society (http://www.thecams. org). The conference was held for the second time in 2013, having been organized before in Jinan in 2011.

P. A. Lieberzeit $(\bowtie)$

University of Vienna, Department of Analytical Chemistry,

1090 Vienna, Austria

e-mail: Peter.Lieberzeit@univie.ac.at
Chemical Monthly has agreed to be one of the journals to publish selected topics presented at ICAM 2013 to an international audience. After peer review, the 34 papers collected in this issue remained. They cover a very broad range of topics with slight emphasis on analytical applications, be it materials analysis or the use of materials science for improving analytical apparatus. Here, special attention is paid to the design of chemical sensors. This is in a way natural, because sensors aim at providing goodvalue analysis, albeit for exactly defined analytical tasks. Generally, the papers clearly represent the shift of materials science from dealing with somewhat "classical, inorganic" solid-state discussions towards both organic structures and the very dynamically developing field of nanotechnology. Both of course allow for tailoring material functionalities on the way to so-called smart materials. In line with this discussion on functionality is the increasing interest in materials synthesis inspired directly by nature, i.e., biomimetic approaches.

It has been a great pleasure and honor for me to act as a guest editor for this issue of Chemical Monthly. I hope that all readers find these papers as enjoyable and useful as the participants at the conference. In addition, I also want to thank the editorial board of Chemical Monthly for their constant support in bringing this issue together. I would be especially pleased if it would motivate some readers to attend the 3rd International Congress on Advanced Materials, which is planned for 2015 and will be announced in due time. 